\title{
Type-2 Fuzzy Extended Kalman Filter for Dynamic Security Monitoring Based on Novel Sensor Fusion
}

\author{
Tarek Dakhlallah ${ }^{1}$, Mohammed Zohdy ${ }^{1}$, Omar Salim $^{2}$ \\ ${ }^{1}$ School of Engineering and Computer Science, Oakland University, Rochester, USA; ${ }^{2}$ Electrical Engineering Department, Benha \\ University, Benha, Egypt. \\ Email: Tkdakh12@oakland.edu, zohdyma@oakland.edu, omar.salem@bhit.bu.edu.eg
}

Received October $19^{\text {th }}, 2011$; revised March $16^{\text {th }}, 2012$; accepted March $23^{\text {rd }}, 2012$

\begin{abstract}
In this paper, we have focused on several relevant sensors [Laser (for speed measurements), Sonar (for space scanning) and RF (for access rights)] to cooperate in monitoring the security status of multiple dynamic agent in surveillance area. Such coordination is achieved by employing novel concepts of sensors similarity and complementarity. Furthermore, this system is aided with Extended Kalman Filter (EKF) in order to estimate the agent's non-linear movement. Finally, transforms system state to be able to make a security suspiciousness decision by using type-2 fuzzy logic system to handle uncertainty. It is shown that the system performance can exhibit promising improvements for this dynamic security monitoring application.
\end{abstract}

Keywords: Sensor Similarity; Sensor Complementarity; Type-2 Fuzzy

\section{Introduction}

Sensors connect the gap between environment under observation and the actual measurement. They form the most important part of outputs of interest in the environment. Unreliable and improperly used sensor readings will result in wrong determination and inappropriate subsequent decisions [1].

Data fusion in general combines data of different sources in order to achieve inferences. For example, while a groundfighter is unable to see around hidden corners or through a tree-dense area [2], additional sensory sources can provide advanced alarm. Similarly, it may not be possible to determine the quality of one kind of food based merely on the sense of taste, but edibility may be arrived at using a combination of vision and smell. Multi-sensor data fusion is naturally performed by animals and humans to achieve more accurate evaluation of the surroundings and identifying dangers, where the objective is increasing their chances of survival [3].

Measurement data may be merged (fused) at different levels, at observation level; and at the decision level. Raw sensor data can be directly combined by similarity if the sensor data are homogenous [3]. There also has been increasing interest in making distributed sensor-based security systems. It is essential to understand how moving objects interact with each other and the environment to extract the major parameters for the development of automated situational security system [4]. In addition to the issue of automated situational awareness, privacy protection is another important issue in monitoring. It is very desirable for a surveillance system to recognize human activities.

Successful implementations of many commercial and military applications require reliable, timely, and precise information to support decisions for remote security operations. Developing effective security monitoring mechanisms to provide situation awareness has become an increasingly important focus. Thus, relying on raw senor data is extremely challenging primarily because security events change continuously and security space information is usually incomplete and noisy. Some dynamic security monitoring systems combine a number of different techniques to data collected from distributed sensors like intrusion detection based on fusing decisions and information correlation to compute event indicators [5].

This paper presents a methodology for multi-sensor based analysis of a surrounding of a highly valuable asset. This is an essential part of a decision making system of an awareness system [6]. Multi-Sensor based networks are an emerging technology that promises ability to monitor the world. The aim of the work in this paper is to be able to estimate (predict) the next security status of a moving agent in an indoor monitored space and be able to make a decision on the system status at any given time $\mathrm{t}$. 
Section 3 below provides a detailed introduction of the proposed security system.

\section{Sensor Types}

This proposed system utilizes three types of sensors to accomplish its goal. Sonar sensor [7] to scan space for total number of moving agents, laser sensor [8,9] to measure speed of agent and finally radio frequency sensor $[10,11]$ to provide the system with agent identification.

Sonar sensors are mainly categorized into propagation and distance types. The LV MaxSonar-EZ0 [7] is one type of those sensors that can be utilized for such application capable to cover up to 254 inches of distance and makes a reading every $50 \mathrm{msec}$ and its cone diameter is wide enough to completely cover the floor part of the area of interest. This set of sensors is responsible to report the total number of the existing agents in the area under surveillance.

On the other side laser sensors [8,9] are also grouped under two major types that are displacement and position. The CSI-430 [8] sensor is capable to capture moving agent's speed up to 30 feet away and it provides the system with resolution feedback with a reading that is 5 digits.

Finally, Radio Frequency sensors [10,11] utilize radio waves propagation to transfer data. The Tag-it HF-I [10] sensor set that is equipped with $13.56 \mathrm{MHz}$ transponders could be used to acquire and report the access right of any agent that enters the space of interest.

\section{A Monitoring System}

In a target monitoring applications; multi-sensor data usually transferred to measurements of angular direction and range which in turn fed into an estimator to estimate the target's next position and velocity (system states) utilizing measurements from different sensors. Similarly, measurements of the target's different attributes and analyzing the motion type of the target with respect to a reference point, helps in making a decision of the intent of the target (e.g., flag and alarm or no-flag needed). The determination of the target's next position and velocity from a noisy time-series of sensor data forms a typical estimation problem where Extended Kalman filtering techniques fits best [3].

This paper proposes a new monitoring system model used to predict the next state of moving agent(s) in a closed space as in Figure 1 by fusing information from multiple sensors of different types.

The area under surveillance is divided into four zones A, B, C and D shown in Figure 1 where each is only a ring with a width that is wide enough to be completely covered by the sonar sensor's cone diameter. This sensor

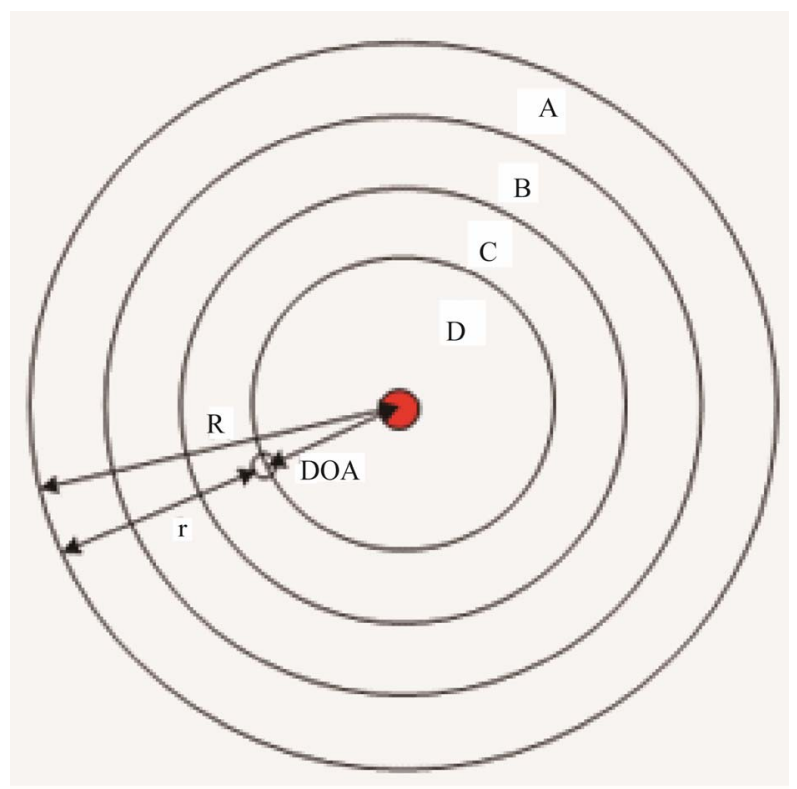

Figure 1. Security monitoring system space with the red circle denotes the valuable asset.

could be mounted in the middle of the ceiling of each ring and rotating at a fixed scanning speed to provide a total number of agents at any given time in any zone [6,7]. As shown in Figure 2 the model also reads in data from a grid of laser sensors $[6,8,9]$ to capture agent speed. The following laser sensor network was assumed; four sensors in the $\mathrm{X}$ direction and another set of four laser sensors in the $\mathrm{Y}$ direction with each of them reporting the agent(s) speed in feet per second as in Figure 2.

Finally an identification data transmitter is associated with each agent and captured by RF Radio Frequency sensor $[10,11]$ to support the system with an ID of any moving agent. For sake of simplicity, Radio Frequency sensor will provide three pre-defined types of agent's access rights ("Trusted", "Semi-Trusted" and "Unknown"). Laser and sonar sensor data sets will then be fed into a sensor similarity processing sub-system that will be responsible to filter out any noisy sensor input of

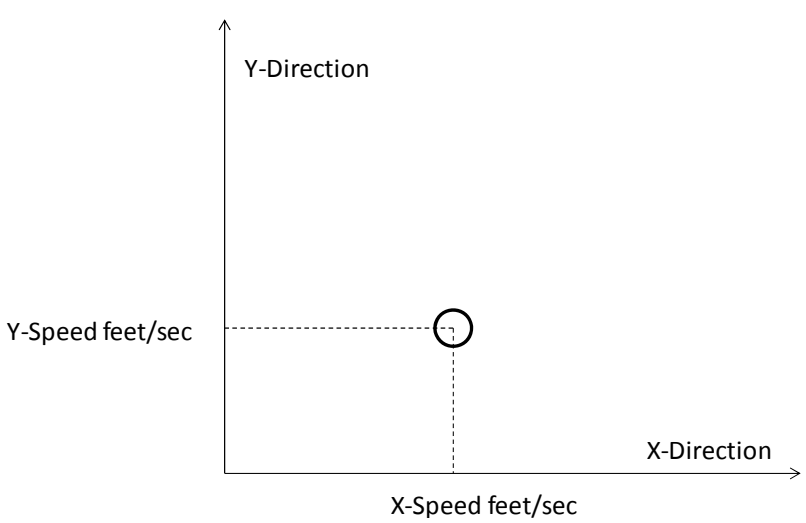

Figure 2. Agent speed captured on $\mathrm{X}$ and $\mathrm{Y}$ direction. 
each sensor-type and come up with a single reading based on sensor similarity method. After sensor data have been filtered, speed on $\mathrm{X}$-axis and Y-axis outputs will be processed in a state estimation and transformation.

Finally sensor complementarity stage starts where sensor data fusion/complementarity is performed using type-2 fuzzy logic inference system to produce a suspiciousness decision for each moving agent as shown in Figure 3.

\section{System Framework (Sensor Similarity)}

Considering raw sensor measurements into sensor fusion may affect quality of fusion which leads to making wrong decisions in some cases where these measurements contain noisy and inaccurate data. Therefore, preprocessing of this sensory data plays an important role in sensor fusion. Only reliable subsets of the sensors are needed; subsets that are consistent and accurate.

In our proposed system data is collected from a total of eight different laser sensors mounted on the $\mathrm{X}$-axis and on the $\mathrm{Y}$-axis (four sensors on each axis) with each sensor having different standard deviation and mean. This paper proposes a new method of sensor similarity that utilizes the concepts of relative closeness of sensors with respect to each other. Over all logic flow of sensor similarity is summarized in Figure 4.

Sensors closeness in measurement between sensors is defined as the distance of sensor " $j$ " with respect to other sensors on the same axis to be:

$$
d_{j}=\left(\operatorname{Max}(j)_{\text {Pos }}-G A_{\text {mean }}\right) / \sigma_{j}^{2}
$$

where $G A_{\text {mean }}$ is the Global mean of all sensors (on a given axis) and $\operatorname{Max}(j)_{P o s}$ is the best estimate of the true state of sensor " $j$ " for data collected over one second time span sensor readings that are closest to the true state and defined as:

$$
\operatorname{Max}(j)_{P o s}=\operatorname{Max}\left[\operatorname{Posteriori}\left(\widehat{x}_{j}\right)\right]
$$

where Posteriori of $j^{\text {th }}$ sensor's reading given the observation $x_{j}$ is $P\left(s_{j} \mid x_{j}\right)$.

\subsection{Iterative Bayes Estimate and Maximum Posteriori}

In this estimate the posterior of the different sensors was obtained based on Bayes formula:

$$
P(s \mid x)=\frac{P(x \mid s) P(s)}{P(x)}
$$

where " $s$ " is the state and " $x$ " is the observation. The probability of " $s$ " given the observation " $x$ "; the observation drawn from normal distribution $\mathrm{N}\left(\mu, \sigma^{2}\right)$ where $\mu$ is the mean and $\sigma$ standard deviation, so the mean of the likelihood function is the state under consideration which is represented as:

$$
P(x \mid s)=\frac{1}{\sigma \sqrt{2 \pi}} e^{\left(-(x-s)^{2} / 2 \sigma^{2}\right)}
$$

and:

$$
P(x)=\frac{1}{\sigma \sqrt{2 \pi}} e^{\left(-(x-\mu)^{2} / 2 \sigma^{2}\right)}
$$

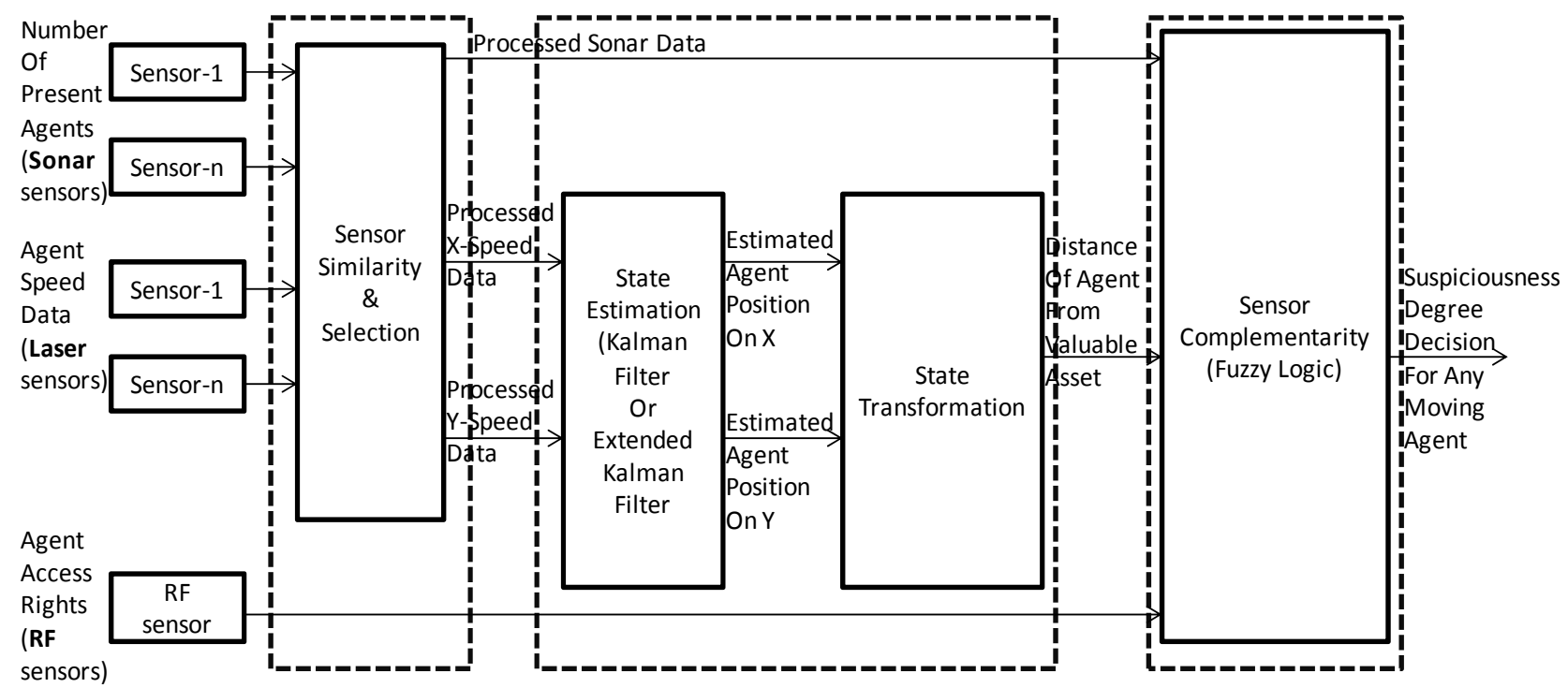

Figure 3. Security system block diagram. 


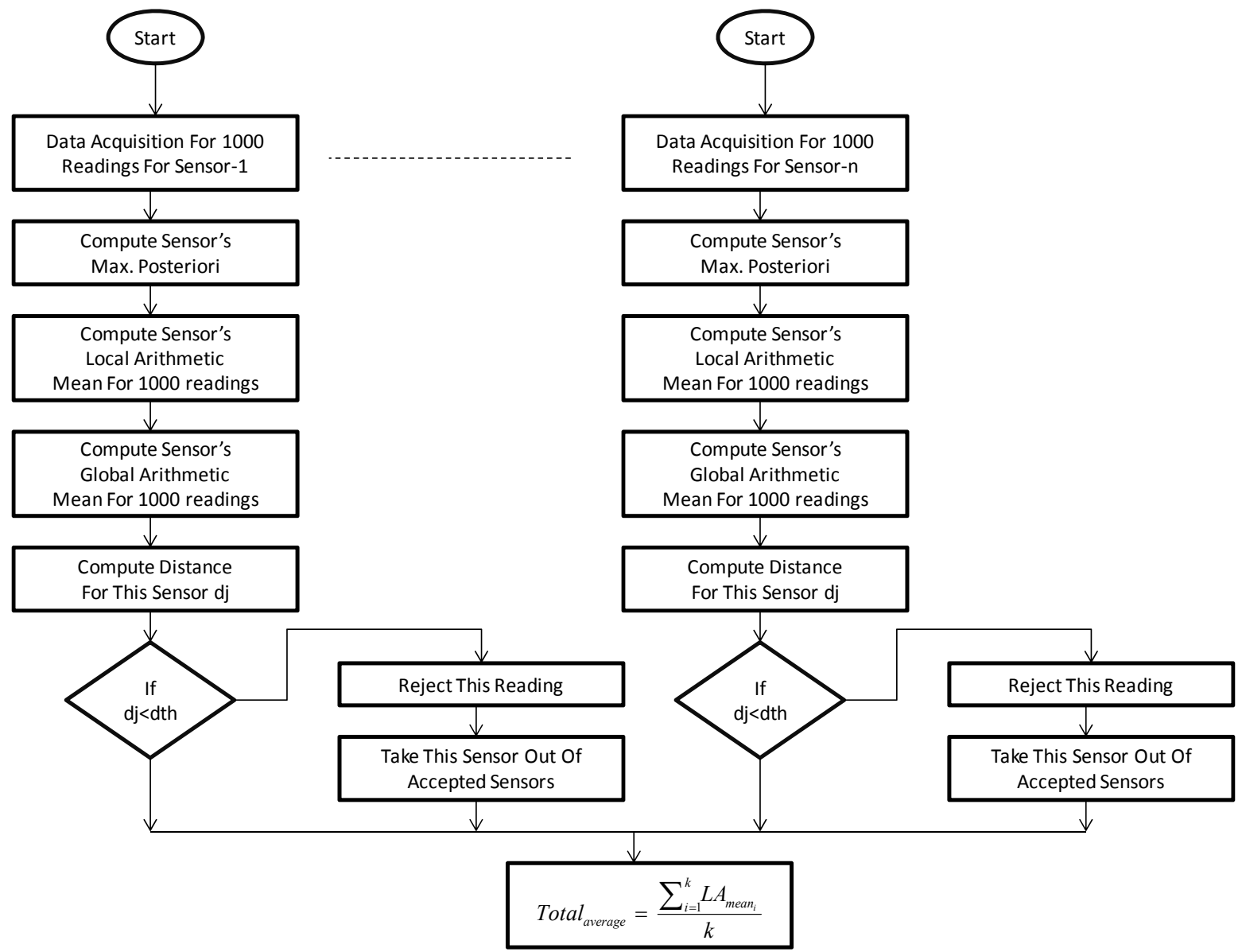

Figure 4. Flow chart shows how sensor similarity is performed.

The only unknown term left in Bayes is $P(x)$. We know that:

$$
\sum P(s \mid x)=1
$$

so:

$$
\sum \frac{P(x \mid s) P(s)}{P(x)}=1
$$

then:

$$
P(x)=\sum P(x \mid s) P(s)
$$

The right side of Equation (8) is computed for all observations and then divided by the total sum of these values to compute $P(x \mid s)$. This process was made iterative as more observations arrive by setting the priori $P_{r}(i+1)=$ Posteriori of the previous observation $P_{0}(i)$ and the maximum posteriori is pulled out at each iteration.

\subsection{Global and Local Means}

In this paper, the $L A_{\text {mean }}$ is defined to be the Local Mean [12] for each sensor over its $k$ observations and defined as:

$$
L A_{\text {mean }}=(1 / k) \sum_{i=1}^{k} x_{i}
$$

and $G A_{\text {mean }}$ in Equation (1) to be the mean of all sensor local means that is defined as follows:

$$
G A_{\text {mean }}=\frac{\sum_{n=1}^{4} L A_{\text {mean }}}{n}
$$

where $n$ is the number of used sensors for an axis ( $n=4$ in our example) and finally, $\sigma$ in Equation (1) refers to the standard deviation of the sensor " $j$ " given the fact that each sensor has a different mean and standard deviation.

After each sensor's $d_{j}$ is calculated it is compared to a pre-defined threshold distance $d_{t h}$ to determine if the reading of this sensor should be rejected or considered. In this paper we assume threshold $d_{t h}$ is concluded from a previously conducted calibration of the sensor network.

If the sensor's reading is considered then it is factored in when calculating the overall average of all considered readings:

$$
\text { Total }_{\text {average }}=\frac{\sum_{i=1}^{n} L A_{\text {mean }_{i}}}{n}
$$


where " $n$ " here is the total number of accepted sensors.

Finally, a single reading as a similarity output is obtained. This algorithm of similarity is applied to laser sensors on both $\mathrm{X}$ axis and $\mathrm{Y}$ axis, and to the sonar sensors as well.

\section{State Estimation and Transformation}

\subsection{Extended Kalman Filter}

Real life dynamic systems and sensors are not absolutely linear, but they are close to be. Thus, applying Extended Kalman filter (EKF) on non-linear problems is a better fit.

Extended Kalman filtering is a method that linearizes about the covariance and current mean $[13,14]$.

Extended Kalman filter uses measurements that are observed over time that contain noise, and produces values that tend to be closer to the true values of the measurements and their associated calculated values. The Extended Kalman filter is a set of mathematical equations that provides an efficient computational (recursive) means to estimate the state of a process, in a way that minimizes the mean of the squared error. Extended Kalman filtering is an ongoing cycle of time updating that projects the current state estimate ahead in time and the measurement updating that adjusts the projected estimate by an actual measurement at that time. The equations for those two updates are presented below [13,14]:

The first step is the Prediction (EKF time update):

1-Project the state ahead:

$$
X_{k}=f\left(X_{k-1}, u_{k}\right)
$$

where $X_{k}$ is the state vector (agent's position and velocity), $A_{k}$ is the state transition model that is applied to the previous state $X_{k-1}, B_{k}$ is the control input model that is applied to the control vector $u_{k}$ and $w_{k}$ is the process noise that is assumed to be drawn from a zero mean normal distribution with covariance $Q$.

$$
X_{k}=f\left(X_{k-1}, u_{k}\right)
$$

2-Project the error covariance ahead:

$$
P_{k}^{-}=A P_{k-1} A^{T}+Q
$$

The second step is the update (EKF measurement update):

1-Compute the EKF gain:

$$
K_{k}=P_{k}^{-} H^{T}\left(H P_{k}^{-} H^{T}+R\right)^{-1}
$$

where $H$ is the measurement vector of the measurement $z_{k}$ of the true state space:

$$
z_{k}=h\left(x_{k}\right)+v_{k}
$$

$v_{k}$ is the measurement noise that is assumed to be drawn from a zero mean normal distribution with covariance $R$.

2-Update estimate with measurement $z_{k}$ :

$$
x_{k}=x_{k}^{-}+K_{k}\left(z_{k}-h\left(x_{k}^{-}\right)\right)
$$

3-Update the error covariance:

$$
P_{k}=\left(I-K_{k} H\right) P_{k}^{-}
$$

For application purposes, estimates of the agent's position on the $\mathrm{X}$ and the $\mathrm{Y}$ axes of the space are needed. Extended Kalman Filter was chosen to accomplish this task.

A system state was defined in this case to be the position and the speed.

$$
\begin{gathered}
V_{x}=X_{-} \text {Total }_{\text {average }} \\
V_{y}=Y_{-} \text {Total }_{\text {average }}
\end{gathered}
$$

where the agent's speed at the $\mathrm{X}$-axis is the final $X_{\text {Total } \text { verage }}$ that was arrived at. After collecting agent's data on $X$ axis and deriving its corresponding position data as (same applies for the $Y$ axis):

$$
\begin{aligned}
& P_{x}=t * X_{\text {Total }_{\text {average }}} \\
& P_{y}=t * Y_{\text {Total }_{\text {average }}}
\end{aligned}
$$

where, $t$ is the time.

Extended Kalman filter was applied on our system to estimate agent's next position.

\subsection{State Transformation (Homogeneous Sensor Complementarity)}

Multi-sensor complementarity is the synergistic use of the information provided by different sensory devices to assist in the accomplishment of a system task. It refers to any stage in the integration process where there is an actual combination of different sources of sensory information into one sensory representation [15]. Sensor complementarities or correlation is especially advantageous when heterogeneous sensors are employed because of the potential to aggregate different views of the same incident.

For our application we are interested in the distance of an agent $(D o A)$ from the valuable asset under surveillance; thus a transfer of the system state (agent's position on $\mathrm{X}$ and $\mathrm{Y}$ axes) to another form is needed where it describes the agent's distance with respect to asset. Since the agent could be moving in any direction in the space (its motion angle from asset will always change) we are always interested in that continuously changing distance. Therefore, we need to transform the $\mathrm{X}$ and $\mathrm{Y}$ to $r$ and $\theta$ (Cartesian to polar). This is defined as homogeneous sensor complementarity as the system used reading from multiple sensors of the same type (speed on $\mathrm{X}$ and speed on Y). However, the system will only utilize the $r$ (radial distance) part of that information then $D o A$ is easily cal- 
culated to be $R-r$; where $R$ is the radius of the largest circle where the agent is first detected by the sensors (Figure 1).

\section{Decision Making/Type-2 Fuzzy (Hetrogeneous Sensor Complementarity)}

The last part of this proposed system is the security decision making which utilizes the previous sub-system output to make a decision using an interval type-2 fuzzy system since it is suitable to make a precise decision in uncertain circumstances.

\subsection{Interval Type-2 Fuzzy Inference System}

Unlike a type-1 set where the membership grade is a crisp value in [0,1], a type-2 fuzzy set shown in Figure 5 is characterized by a fuzzy membership function, where the membership of each point of this set is a fuzzy set in $[0,1][16]$.

An Interval type-2 fuzzy set makes room for non-deterministic truth degree and uncertainty [17,18] (foot print of uncertainty FOU shown in Figure 5) for an element that belongs to a set. A type-2 fuzzy set denoted by, $\tilde{A}$ is characterized by a type- 2 membership function, $\mu_{\tilde{A}}(x$, $u)$, where $x \in X, u \in J_{x}^{u} \subseteq[0,1]$ and $0 \leq \mu_{\tilde{A}}(x, u) \leq 1$.

$$
\begin{gathered}
(\tilde{A})=\left\{\left(x, \mu_{\tilde{A}}(x)\right) \mid x \in X\right\} \\
(\tilde{A})=\left\{\left(x, u, \mu_{\tilde{A}}(x, u)\right) \mid \forall x \in X, \forall u \in J_{x}^{u} \subseteq[0,1]\right\}
\end{gathered}
$$

It is the bounded area in Figure 5 and mathematically it is the union of the upper and lower membership functions $[14,18]$, where the upper and lower memberships are Gaussian functions:

$$
\begin{aligned}
& \operatorname{Upper}(\operatorname{FOU}(\tilde{A}))=N\left(m, \sigma_{2} ; x\right) \\
& \text { Lower }(\operatorname{FOU}(\tilde{A}))=N\left(m, \sigma_{1} ; x\right)
\end{aligned}
$$

where, $\sigma_{1}$ and $\sigma_{2}$ are the standard deviations for lower and upper membership functions respectively and $m$ is the mean of both.

\subsection{Heterogeneous Sensor Complementarity}

In this paper a decision making sub-system (an interval type-2 fuzzy logic system) is defined to be the heterogeneous sensor [19] complementarity as it reads in three different sensor data types and generates a fourth type of data that is totally different from the input ones. The proposed security sub-system will generate a percentage output based on the number of agents and their relative distances from the valuable asset at any given time. The first input which is the "Number of Agents" $(\mathrm{NoA})$ is considered an input with four different fuzzy ranges. Ranges are "Low" indicating the total number of objects is in the low scale of the alarming system, a "Medium Low" is the next level up, "Medium High" is the second highest level and finally "High" is the highest possible level. The second input is $D o A_{f}$ that is generated as:

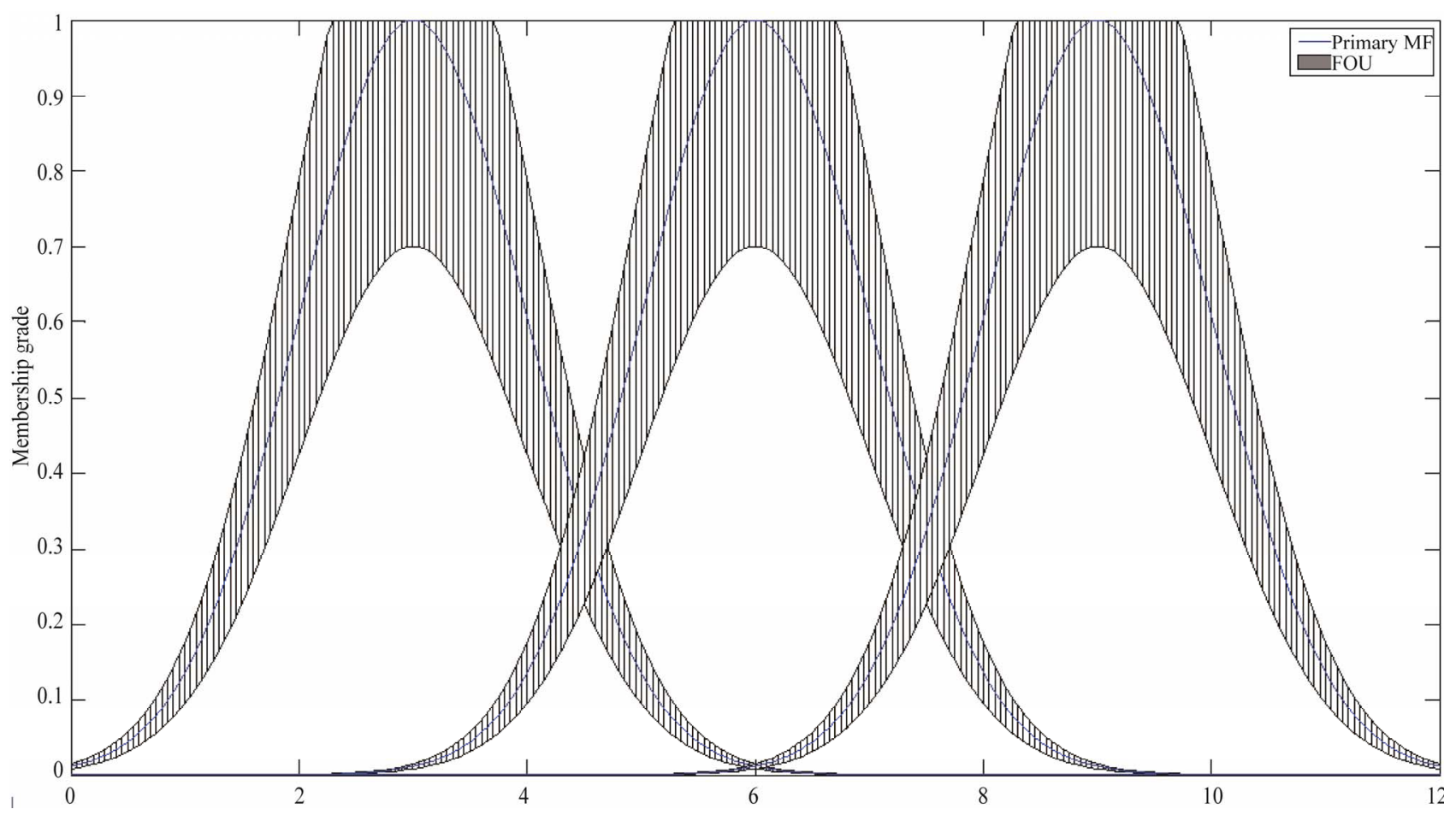

Figure 5. A foot print of uncertainty of a sample interval type-2 Gaussian membership function. 


$$
D o A_{f}=D o A * f
$$

where $(D o A)$ "Distance of Agent" from the asset in feet. $D o A$ is categorized into four different fuzzy categories, "Agent is extremely close", "Agent is very close", "Agent is close" and "Agent is far" (Figure 1). Also, where " $f$ " is a multiplication factor that is based on the "Access Rights" of any moving agent and defined to be twenty for a "Trusted" (denoted by "T"), ten for "SemiTrusted" (denoted by "ST") and finally a one for an "Unknown" agent (denoted by "U”).

The proposed system has only one output which is the "Degree of suspiciousness" $(D o S)$. This $D o S$ is categorized into five levels of suspiciousness, "Not suspicious", "Almost Suspicious", "Suspicious", "Very Suspicious", and "Extremely suspicious" as shown in Figure 6 and is driven by the $D o A_{f}$ and $N o A$ inputs $[6,20]$.

\section{Experimantal Results and Discussions}

In this study a real-life simulation of several situations of agents moving non-linearly were investigated. Evaluation for a one agent is shown here. Assuming agent's true speed is $3 \mathrm{feet} / \mathrm{sec}$ on X-axis the grid of four sensors (each sensor is slightly different from the other in its mean and standard deviation) captured this speed over one second time frame. Figure 7 shows agent speed captured by four sensors.

Sensor 1 of this group is assumed to be noisier with mean and standard deviation well distant from the other three. We also assumed the agent's true speed is 5 feet/sec on Y-axis, the grid of four sensors (each sensor is slightly different from the other in its mean and standard deviation) on this axis also captured this speed over same time interval that is 1 second Figure 8 shows this agent's data on the Y-axis. Sensor 2 of this group is assumed to be the noisy sensor.

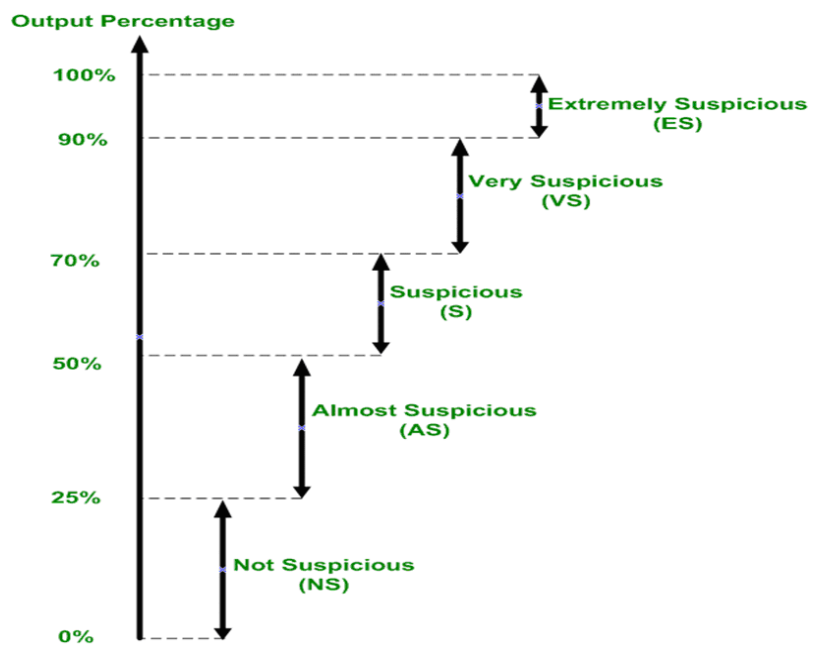

Figure 6. System suspiciousness levels.
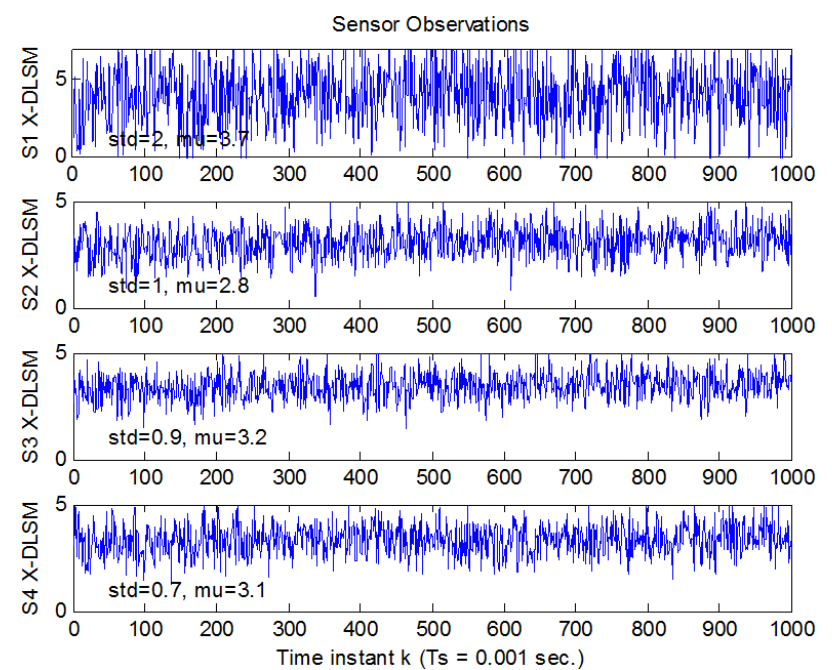

Figure 7. Speed data read by four laser sensors on the $\mathrm{X}$-axis of the sensor grid (where DLSM is Direction Laser Speed Measurement) and the number prefix refers to the sensor index in the grid.
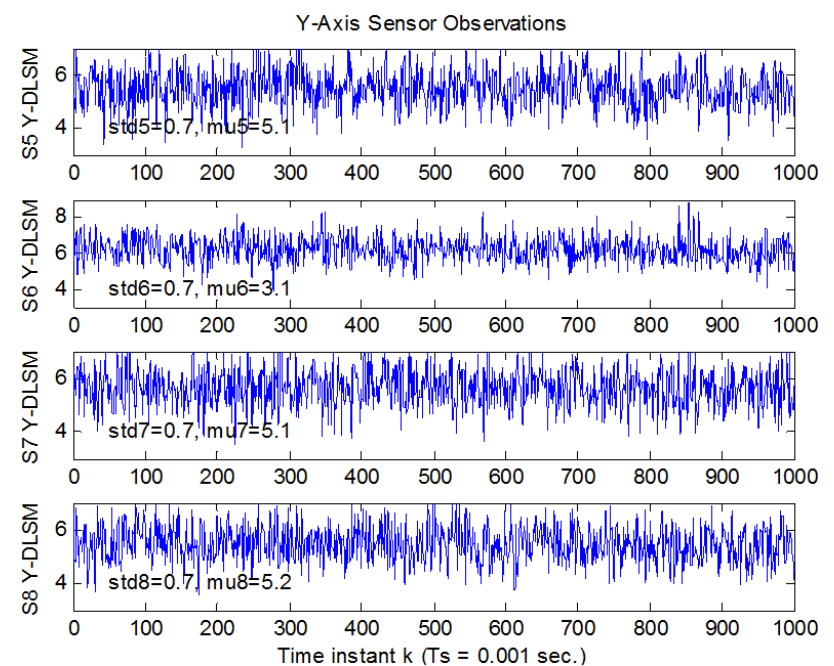

Figure 8. Speed data read by four laser sensors on the $\mathrm{X}$-axis of the sensor grid (where DLSM is Direction Laser Speed Measurement) and the number prefix refers to the sensor index in the grid.

Local means were next computed for each the four sensors on $\mathrm{X}$-axis over for measurement over one second are shown in Figures $\mathbf{9}$ and $\mathbf{1 0}$ show their equivalents on the Y-axis for the same time span. The next step in our sensor similarity is to compute the maximum posteriori for each of the $\mathrm{X}$-axis sensors which are displayed in Figure 11 and compute those posteriori for the $\mathrm{Y}$-axis as well as shown in Figure 12. After sensor similarity is applied for both sets of laser sensors (X and $\mathrm{Y}$ axes), a distance for each sensor was calculated and compared to a pre-defined threshold values that are 0.5 and 0.9 for $\mathrm{X}$ and $\mathrm{Y}$ respectively. 

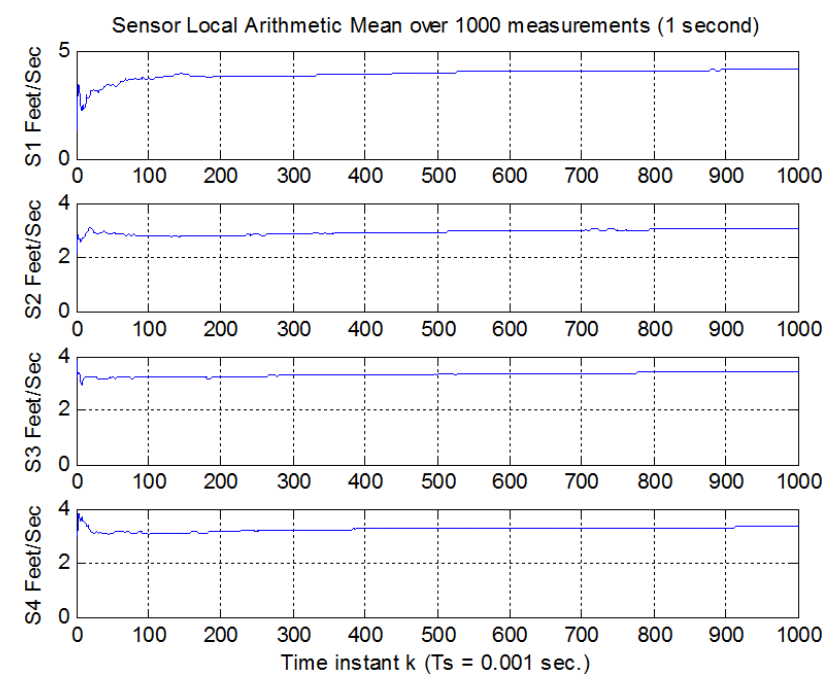

Figure 9. Local mean $\left(L A_{\text {mean }}\right)$ for the four sensors on the $\mathrm{X}$-axis over 1 second time span.
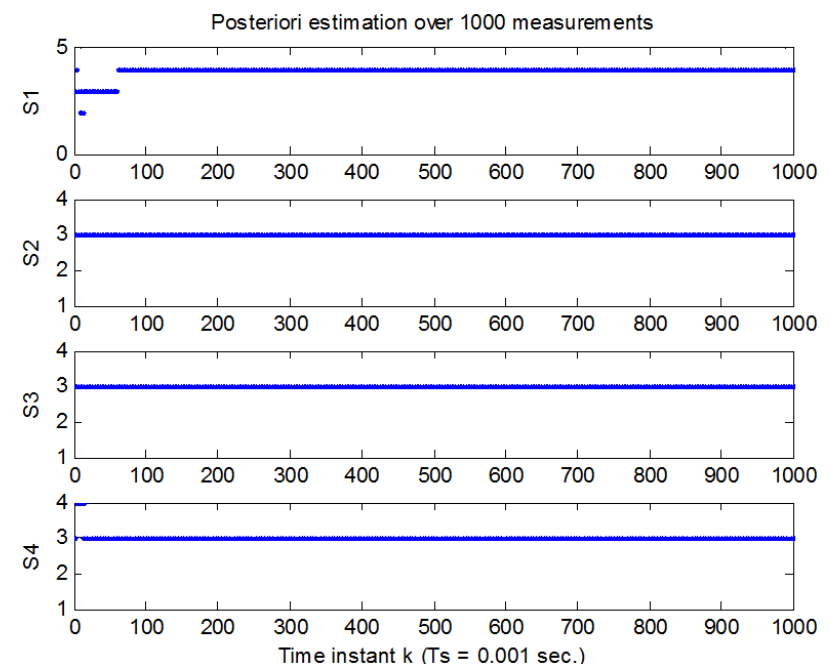

Figure 11. Max. posteriori $(\operatorname{Max}(j)$ pos) for the four sensors on the $\mathrm{X}$-axis over 1 second time span.

Those thresholds are assumed to be based on sensor calibration data for each axis. New global mean Total $_{\mathrm{av}-}$ erage) was computed but based on only accepted sensors. Next Extended Kalman filter was used to estimate agent's next state (next accumulated distance) on both axes based on the $X$-Total ${ }_{\text {average }}$ and $Y$-Total $l_{\text {average }}$ that are shown in Figures 13 and 14 respectively.

State estimation (S.T.) that is the second to last block in our security system is then utilized to transform the distance data on $(x, y)$ coordinates to $(r, \theta)$ polar coordinates (homogeneous sensor complementarity) as in Figure 15.

Finally, a weighing is applied to this computed $r$ (based on its access right that is chosen to be 1.3 and 1.1 for "Trusted" and "Semi-Trusted" respectively and 1 for "Unknown"). Then it is fed to the decision making system to
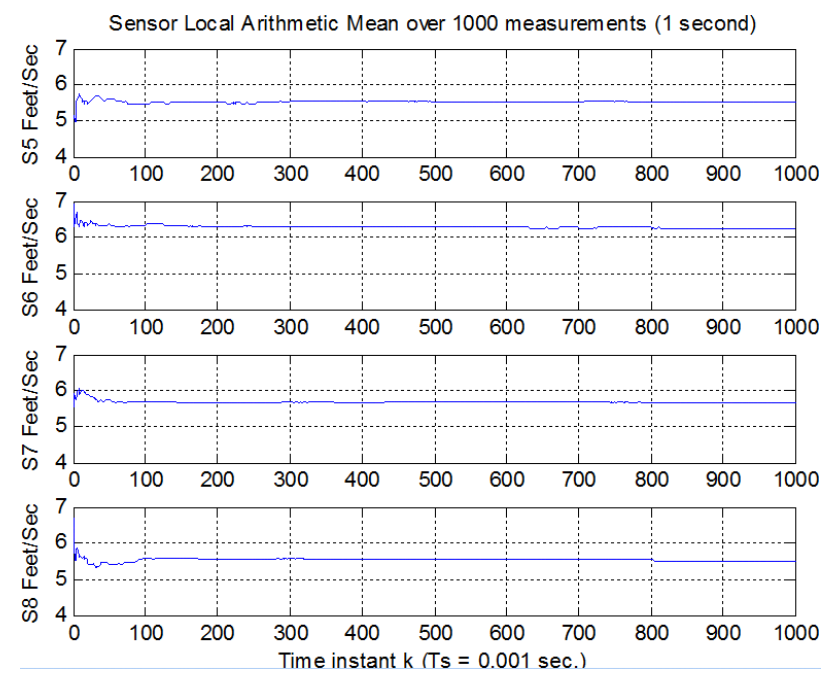

Figure 10. Local mean $\left(L A_{\text {mean }}\right)$ for the four sensors on the Y-axis over 1 second time span.
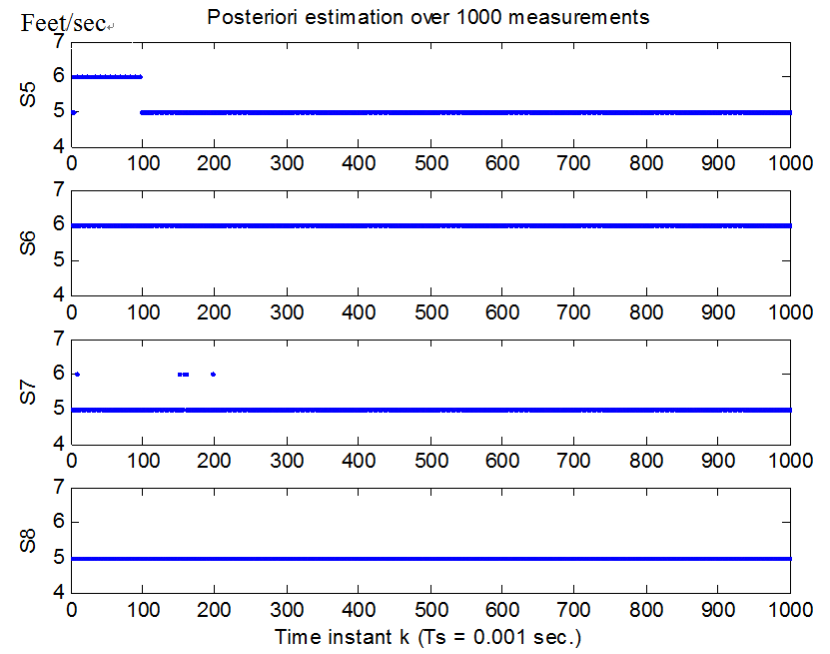

Figure 12. Max. posteriori $(\operatorname{Max}(j) p o s)$ for the four sensors on the $\mathrm{Y}$-axis over 1 second time span.

decide on its suspiciousness degree at any time during its movement shown in Figure 16. This figure displays evaluation two agents having the same speed values but different access rights ("Trusted" and "Unknown"). Figure 16 shows how the proposed security system was able to limit the $D o S$ for the "Trusted" agent to less than $55 \%$. However, it gave the "Unknown" agent almost a $65 \%$ for the same speed and distance accumulated values. It was shown that our system can actually use normal sensor data to filter it, estimate state, transform state and make a decision.

\section{Conclusions and Future Work}

Applying novel sensor similarity and complementarity (homogeneous and heterogeneous) concepts in this paper has helped improving the performance of a dynamic 


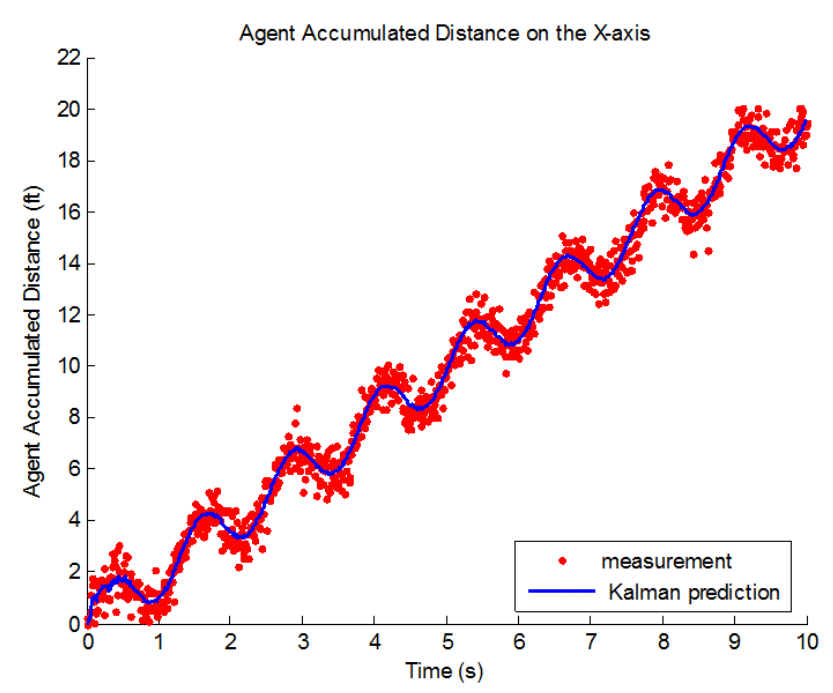

Figure 13. Agent position on $X$ axis where " 0 " is the point where agent was first detected by the sensor.

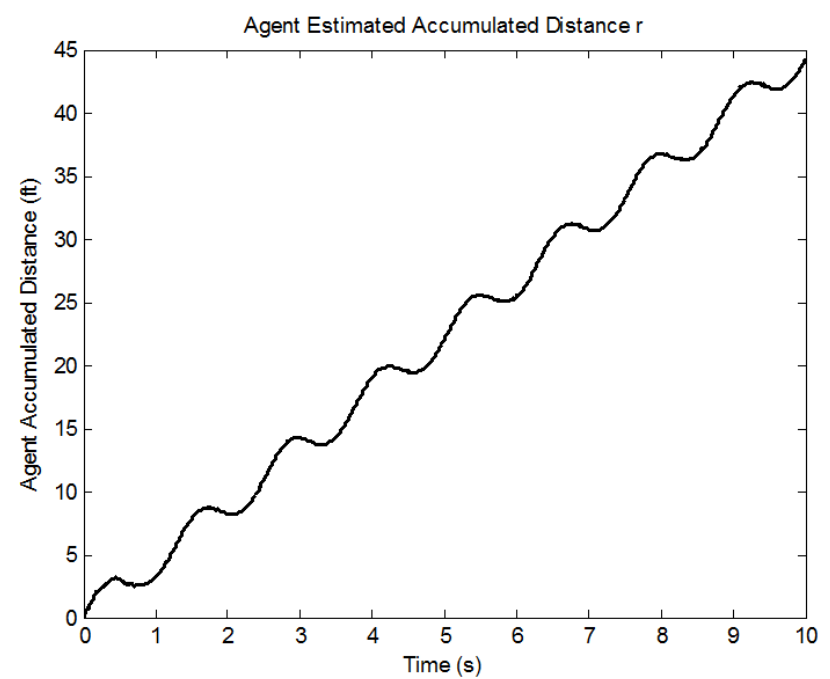

Figure 15. Agent accumulated distance in polar coordinates.

security monitoring system. With the Extended Kalman filter and interval type-2 fuzzy inference help, the system was further able to estimate agent's next state and report its security status. The proposed system exhibits promising performance in security awareness systems and agent security status evaluation accounting for dynamics, non-linearity and uncertainty.

One planned future system improvement is to introduce active relationship between multiple agents and environment.

\section{Acknowledgements}

Authors would like to thank Prof. O. Castillo for providing us his own made type-2 fuzzy toolbox [19], his help is much appreciated.

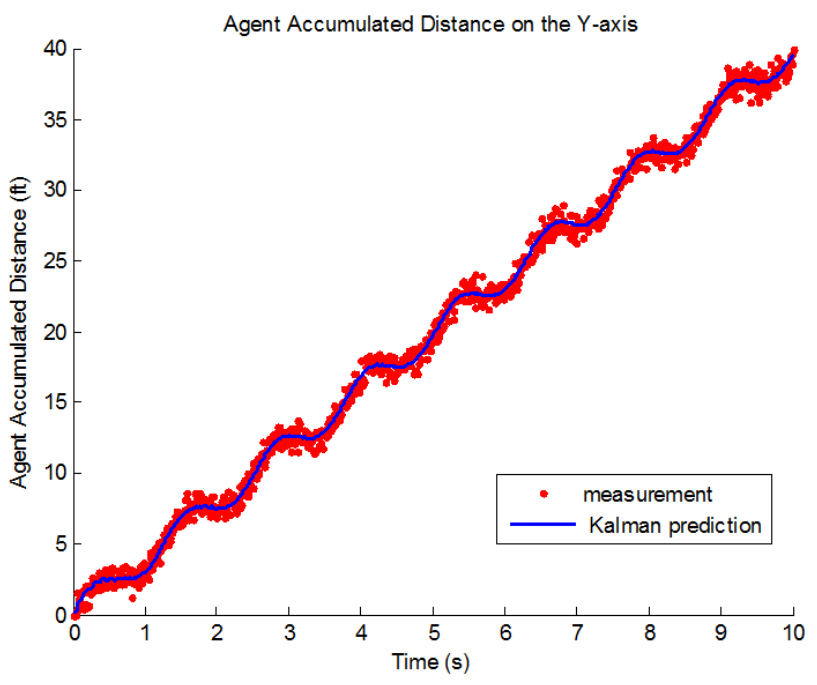

Figure 14. Agent position on $Y$ axis where " 0 " is the point where agent was first detected by the sensor.

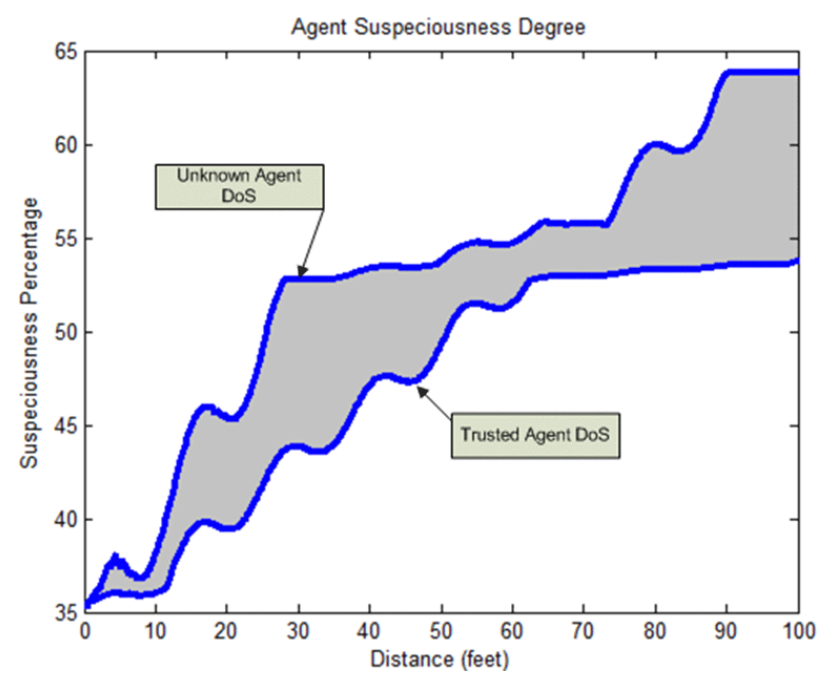

Figure 16. Agent suspiciousness degree as it moves.

\section{REFERENCES}

[1] M. Zohdy and A. Khan, "Global Optimization of Stochastic Multivariable Functions," American Control Conference, San Francisco, 2-4 June 1993, p. 2339.

[2] H. Hujun and Z. Yaning, "Multi-Source Data Fusion Technology and Its Application in Geological and Mineral Survey," 2010 2nd International Conference on Information Engineering and Computer Science (ICIECS), Wuhan, 25-26 December 2010, pp. 1-6.

[3] D. L. Hall and J. Llinas, "An Introduction to Multisensor Data Fusion," Proceedings of the 1998 IEEE International Symposium on Circuits and Systems, 31 May-3 June 1998, pp. 537-540.

[4] Q. Wu, D. Ferebee, Y. Lin and D. Dasgupta, "An Integrated Cyber Security Monitoring System Using Correlation-Based Techniques," IEEE International Conference on System of Systems Engineering 2009, Albuquerque, 30 
May-3 June 2009, pp. 1-6.

[5] R. Tenney and N. Sandell, "Detection with Distributed Sensors," IEEE Transactions on Aerospace and Electronic Systems, Vol. AES-17, No. 4, 1981, pp. 501-510.

[6] T. Dakhlallah, M. Zohdy and O. Salim, "Application of Hyper-Fuzzy Logic Decisions for A Security Monitoring System," 2011 3rd International Conference on Computer and Automation Engineering (ICCAE 2011), 2011, p. V1-387.

[7] LV-MaxSonar-EZ0, "High Performance Sonar Range Finder,” MaxBotix Inc., 2005.

[8] CSI 430, "SpeedVueTM Laser Speed Sensor," Emerson Process Management, 2009.

[9] MiniVLS nL, "Series Optical Speed/Phase Sensors VLS $\mathrm{nL}$," Compact Instruments.

[10] Tag-it HF-I Standard, 13.56 MHz, "Transponder Inlays, ISO/IEC 15693 and ISO/IEC 18000-3 Global Open Standards," Texas Instruments, 2005.

[11] Smart Card SCC-3, “3.125KHz+UHF EPC GEN2," Rui Yue RFID Co., 2006.

[12] Albert Leon-Garcia, "Probability and Random Processes for Electrical Engineering," 2nd Edition, Pearson/Prentice Hall, Upper Saddle River, 2008.

[13] G. Welch and G. Bishop, "An Introduction to the Kalman Filter," University of North Carolina at Chapel Hill, Chapel Hill, 2001.

[14] E. Ayachi, S. Ihsen and B. Mohamed, "A Comparative
Study of Nonlinear Time-Varying Process Modeling Techniques: Application to Chemical Reactor," Journal of Intelligent Learning Systems and Applications, Vol. 4, No. 1, 2012, pp. 20-28.

[15] R. C. Luo and C. Yih, "Multisensor Fusion and Integration: Approaches, Applications and Future Research Directories," IEEE Sensors Journal, Vol. 2, No. 2, 2002, pp. 107-119.

[16] O. Castilo and P. Mellin, “Type-2 Fuzzy Logic: Theory and Applications," Tijuana Institute of Technology, Division of Graduate Studies, Vol. 223, 2008.

[17] Q. Liang and J. Mendel, "Interval Type-2 Fuzzy Logic Systems: Theory and Design," IEEE Transactions on Fuzzy Systems, Vol. 8, No. 5, 2000, pp. 535-550.

[18] J. Mendel, "Uncertain Rule-based fuzzy logic systems: Introduction and New Directions," Prentice-Hall, Upper Saddle River, 2001.

[19] H. Youpeng, L. Lin and Z. Yongfeng, "A Hetrogenous Sensors Track-to-Track Correlation Algorithm Based on Fuzzy Numbers Similarity Degree," Second International Conference on Information and Computing Science, 2009 Manchester, 21-22 May 2009, pp. 191-194.

[20] J. R. Castro, O. Castillo and P. Melin, "An Interval Type-2 Fuzzy Logic Toolbox for Control Applications," International Conference on Fuzzy Systems, London, 23-26 July 2007, pp. 1-6. doi:10.1109/FUZZY.2007.4295341 